\title{
Aportes de la sociología al estudio de la educación (Autores clásicos)
}

\author{
Contributions to the Study of Sociology of Education (Classical authors)
}

\author{
Alexis Rojas-León ${ }^{1}$ \\ Consultor y docente universitario \\ Universidad de Costa Rica, Sede del Atlántico \\ Cartago, Costa Rica \\ alexisrojasleon@yahoo.com
}

Recibido: 22 agosto 2012 Aceptado: 13 marzo $2014 \quad$ Corregido: 25 marzo 2014

Resumen: Desde sus inicios, la sociología se ha enfocado en el estudio de áreas específicas como lo es la educación, hasta convertirse en una ciencia que profundiza en el estudio científico y especializado del tema. Este documento presenta un repaso de los aportes de diversas tradiciones teóricas europeas acerca de la educación. De los "insumos teóricos" presentados, se desprende la reflexión y la propuesta teórica acerca del objeto de estudio de la sociología de la educación, debido a que en ocasiones parece no haber claridad ni consenso entre los autores. Lo anterior con el fin de analizar aquellos aspectos medulares acerca de la comprensión de las múltiples interacciones entre sociedad-educación y viceversa, visibles desde la sociología. Por último, como una de las principales conclusiones, se argumenta que la tradicionalmente llamada sociología de la educación, engloba la sociología educacional, la sociología educativa y la sociología del sistema educativo. Estas constituyen lineas de investigación de un mismo tema, pero su categorización facilita la especificación de diversos aspectos dentro de un mismo objeto de estudio.

Palabras Claves: Sociología, educación, sistema educativo, sociedad.

\begin{abstract}
Since its origins, sociology has focused on the study of specific areas such as education, in order to become a science that not only explores the scientific studies, but is also specialized on the subject matter. This article presents an overview of the contributions of various theoretical European traditions regarding education. From the "theoretical inputs" presented, the reflection and theoretical proposal about the subject matter related to the sociology of education are re-defined, due to a seemingly lack of clarity or consensus among the authors. Mainly, this research attempts to analyze those core issues related to the understanding of the multiple interactions between society-education and vice versa, visible from sociology. As one of the main findings, it is argued that the traditionally named sociology of education encompasses three subject matters: sociology of education, pedagogical sociology and the sociology of the educational system. Although these lines of research are intertwined, its categorization facilitates the specific understanding of various aspects within the same subject matter.
\end{abstract}

Keywords: Sociology, education, education system, society.

\footnotetext{
Sociólogo, educador e historiador (UCR), ha sido docente universitario en educación (UCR-Sede del Atlántico), en tesis de graduación, métodos de investigación e historia para las carreras de Publicidad y Derecho (Universidad Internacional de las Américas); teoría social para Enfermería y Trabajo Social (Universidad Latina); consultor para el Colegio Federado de Ingenieros y de Arquitectos (CFIA); docente en estudios sociales y educación cívica en colegios públicos y privados; investigador del Instituto de Investigaciones Sociales (IIS-UCR), del Centro de Investigaciones Históricas de América Central (CIHAC) y consultor para Programa de las Naciones Unidas para los Asentamientos Humanos (ONU-HABITAT).
} 


\section{Introducción}

Al analizar el desarrollo de la sociología como ciencia social, desde la época que se remonta a los autores clásicos hasta la actualidad, se puede observar que la comunidad científica sociológica ha mostrado un especial interés en la aplicación del conocimiento sociológico al estudio de la educación desde diversas perspectivas.

No es de extrañarse que el desarrollo de la llamada «sociología de la educación» tenga un fuerte impulso en Europa, el mismo continente que vio surgir y consolidarse a la sociología en el seno de la academia desde el siglo XIX.

Esta larga trayectoria ha dado como resultado numerosos aportes teóricos e investigativos que tienen una gran utilidad para quienes pretendan iniciar sus estudios superiores en la rama de la educación.

De esta manera, se presenta una revisión de los aportes que diversas tradiciones teóricas han hecho acerca del tema, así como una reflexión acerca del objeto de estudio de la sociología de la educación. Además, es una contribución al crecimiento de la producción teórica y del conocimiento acerca de la educación, a partir de los planteamientos aquí desarrollados.

$\mathrm{Su}$ objetivo es aportar a la población estudiantil (ya sea de ciencias de la educación o enseñanza de...) que recién comienza su formación en esta área, un documento que facilite la introducción a este campo desde la perspectiva sociológica, mapeando las diversas corrientes, autores y textos teóricos y apuntando algunos de sus planteamientos principales. A manera de ubicación temporal se mencionarán el año de nacimiento y de muerte de los autores clásicos (a saber: E. Durkheim, M. Weber, K. Marx, G. Simmel), y el año de publicación de las obras y de la edición empleada.

Por otro lado, se agradecen los aportes y aclaraciones sobre teoría sociológica del Dr. Óscar Fernández González, quien ha sido un mentor para muchas generaciones de estudiantes en la carrera de sociología (Universidad de Costa Rica), a quien por sus enseñanzas se le debe el estímulo intelectual que requiere profundizar en el campo de la teoría.

\section{Desarrollo del tema}

\section{1. ¿Quée es? Y ¿para qué sirve la sociología?}

Estas preguntas parecen "inocentes", pero son cruciales para entender ampliamente lo que se desarrollará más adelante, debido a que la sociología, según Adorno y Horkheimer (1969), nace como "hija del positivismo" y, desde sus inicios, busca liberar a la voluntad y el conocimiento de la influencia del saber religioso y de cualquier especulación metafísica proveniente de la filosofía, por medio de la rigurosidad y objetividad de la investigación científica aplicada a la comprensión de los fenómenos sociales.

Es decir, si bien la "novedad" de la sociología no se encontraba en su objeto de estudio (la sociedad), siendo este parte de la reflexión humana ya desde épocas anteriores (como en Herodoto, Ibn Jaldun, etc.), lo "novedoso" y el aporte de la sociología son los métodos y las interpretaciones teóricas acerca de la sociedad que surgirán del estudio científico de la misma. 
Así, la sociología, al igual que muchas otras ciencias, comenzó un largo camino de construcción que llevó a un desarrollo de ideas, teorías, métodos, técnicas y debates acerca del objeto de estudio propio de la sociología (Berthelot, 2003), que sirvieron (y sirven) como parte de una constante "revisión" de los logros de la sociología como ciencia.

De esta forma se fue dando un distanciamiento de lo emocional y lo religioso como forma de comprender la realidad, pasando a una racionalidad a través de la ciencia y tratando de evitar el traslado mecánico de conocimientos, técnicas y métodos de las ciencias naturales hacia las ciencias sociales (Elias, 2002), desarrollando métodos y técnicas propios (como toda ciencia) y dándole a la sociología la categoría de conocimiento científico.

A partir de esto, la sociología fue lentamente desarrollando los elementos necesarios de toda ciencia, como lo es el "empleo sistemático y crítico de la investigación, el pensamiento teórico y el pensamiento lógico-argumental para desarrollar un cuerpo de conocimiento sobre un objeto definido" (Taberner, 2005, p. 17).

Pero como señala este mismo autor, el conocimiento científico es diverso, debido a que no todas las ciencias son experimentales. No obstante, la crítica y la aplicación rigurosa del método son requisitos necesarios de la sociología al igual que en toda ciencia.

En relación con el desarrollo del pensamiento teórico dentro del caso específico de la sociología, con el paso del tiempo y la aparición de más personas interesadas en este campo, surge una pluralidad de enfoques teóricos y formas de analizar la sociedad y los fenómenos sociales, dando paso a la aparición de autores que dejaron detrás de sí una larga tradición teórica en la sociología.

Ahora bien, todo lo anterior nos lleva a hacer la pregunta ¿qué es la teoría? Para los fines conceptuales de este documento, se entiende por teoría

Un conjunto de proposiciones acerca de un ámbito de objetos conectados entre sí de tal modo que a partir de algunas de ellas se pueden deducir las restantes [...] su validez real consiste en que las proposiciones deducidas concuerden con acontecimientos efectivos. Por el contrario, si se presentan contradicciones entre la experiencia y la teoría, habrá que revisar una u otra. O bien se ha observado mal, o bien hay algún error en los principios teóricos. De ahí que la teoría permanezca siempre, respecto a los hechos, como una hipótesis (Horkheimer, 2000, p. 23).

Para alcanzar esto, toda teoría debe desarrollar una consistencia y coherencia interna que le permita interpretar los fenómenos sociales (o a la sociedad como un todo), desarrollando conceptos propios y un abordaje capaz de describir, interpretar y explicar su objeto de estudio.

De esta manera, términos como funcionalismo, funcional-estructuralismo, estructuralismo marxista, fenomenología, etc., empezaron a ser de uso común dentro de la sociología, al nombrar la clasificación de las diversas corrientes teóricas que aparecieron durante el siglo XX. Por lo anterior, las respuestas a la pregunta "¿qué es la sociología?" dependerán de la perspectiva o tradición teórica desde la que se posicione un autor.

No obstante, de forma sucinta y simplificada, se puede definir sociología como la ciencia que estudia la sociedad con sus propios métodos, tanto su estructura como la acción social, que 
toma a la sociedad como "un todo" (a partir de sus elementos objetivos) o la visualiza desde la escala de los individuos (subjetivos e intersubjetivos), poniendo el énfasis en la dimensión macrosocial o microsocial respectivamente.

Para esto emplea métodos de investigación cualitativa y cuantitativa dando como resultado el análisis, comprensión y reflexión de los fenómenos sociales y las interrelaciones entre distintos áreas o aspectos sociales.

Es en este punto donde se puede hablar de la sociología como un campo plural, es decir, se trata de reconocer la existencia de «sociologías» que hacen énfasis en unos u otros aspectos pero que al mismo tiempo permiten clasificar la producción sociológica como algo diverso.

Así por ejemplo, se han dado discrepancias entre quienes ven a la sociología desde un punto de vista "empirista" o "teoreticista" (Horkheimer, 2000); quienes hablan de una sociología experimental frente a una sociología social, sociología académica, etc. (Lahire, 2006b); o los debates entre "pragmáticos sociales" y "filósofos sociales" (Adorno, 2006), que se diferencian por sus posiciones (en distintos grados) acerca de la importancia de la evidencia empírica, la construcción de la teoría, la elaboración de las investigaciones, el desarrollo de un abordaje metodológico innovador y exhaustivo, etc.

Habiendo detallado en síntesis en qué consiste la teoría sociológica, surge una nueva pregunta, la cual es ¿para qué sirve la sociología? Es claro que esto lleva a analizar el sentido de utilidad del saber sociológico y su valor académico y extraacadémico, en una ciencia con una función diversa (determinada por quien produce dicho conocimiento) sobre la sociedad y tiempo actual, que por su misma función y diversas perspectivas se encuentra destinada constantemente a ofrecer sus resultados y contestar: “¿¿Para qué sirve realmente tal o cual sociología?" O "icómo los actores sociales utilizan publicaciones sociológicas a las que pueden tener acceso?"” (Lahire, 2006b, p. 28).

Precisamente, la sociedad legítima o deslegitima el saber sociológico según la función que le dé quien investiga y la utilidad que se le asigne socialmente. De esta forma, para algunos la sociología es capaz del análisis y diagnóstico de la sociedad, para otros aporta el ideario de la lucha de los oprimidos en contra del poder, para algunos es la generadora de instrumentos para la dominación de quienes ejercen poder político y para otros, se integra en proyectos de cambios sociales por medio de la acción participativa de comunidades.

Al final la utilización del conocimiento sociológico por parte de la comunidad científica, los actores sociales y los actores políticos encargados de la toma de decisiones importantes, son en última instancia quienes definen la importancia (práctica) de los aportes de la sociología para la sociedad.

Por supuesto, muchas de las investigaciones en sociología tienen trascendencia en el ámbito académico universitario y en el seno de la comunidad científica. Pero, ¿cuánto del conocimiento sociológico producido por las investigaciones realizadas en Costa Rica forma parte del repertorio de conocimientos de los estudiantes de carreras de educación? ¿Cuánto conocen los profesionales en educación que ejercen en primaria o secundaria sobre las investigaciones más recientes? ¿Cuántas de las investigaciones realizadas en los últimos años habrán sido consideradas por quienes ocupan puestos importantes en el Ministerio de 
Educación Pública (MEP) para la toma de decisiones? ¿Por qué en documentos oficiales del MEP no se hace mención de estas investigaciones y de sus resultados?

Desafortunadamente esa es la realidad que enfrenta quien desee ejercer la sociología. Sin embargo, esto no es una "sentencia del destino", sino una realidad que podría cambiar, empezando en la propia comunidad científica de la sociología, la cual necesita revalorar la importancia de su trabajo.

Por otro lado, la simple definición ofrecida por sí misma no permite comprender en toda su extensión a la sociología como ciencia y sus posibles repercusiones en la realidad social. Para lograr esto es necesario profundizar en las diversas perceptivas o tradiciones teóricas.

En cuanto a su utilidad, este es un tema de debate que depende de la posición asumida, tanto de quien pregunta como de quien contesta y que sólo el lector podrá juzgar cuando profundice, por su cuenta, en la producción sociológica.

\subsection{Las tradiciones teóricas relacionadas con el estudio de la educación}

Han sido numerosos los aportes que desde la sociología se han desarrollado acerca de la educación. Tantos aportes que se podrían organizar por escuelas, tradiciones o corrientes para un mayor entendimiento del tema y de las diversas perspectivas dentro de una misma ciencia.

\subsubsection{El Funcionalismo y la influencia de Durkheim}

También conocida por algunos autores como la perspectiva del consenso o positivista, ha tenido una larga tradición dentro de los ámbitos académicos. Aquí particularmente se hará referencia a uno de los principales exponentes en Francia.

En su obra el filósofo Emile Durkheim (1858-1917) le da a la educación y al sistema educativo un lugar prioritario en relación con la sociedad. Junto a su producción escrita, su carrera docente combinó en varias ocasiones la educación y las ciencias sociales.

Lo anterior sucedió cuando obtuvo la cátedra de pedagogía y ciencias sociales de la Universidad de Burdeos (1896), o cuando fue nombrado profesor titular del curso de ciencia de la educación y sociología en la Universidad de la Sorbona (1902), donde "defendió la necesidad de recurrir a la sociología de la educación para sustentar tanto las teorías como las prácticas pedagógicas, pues toda educación es de naturaleza social" (Álvarez-Uría, 2007, pp. 117-118).

$\mathrm{Su}$ perspectiva sociológica prioriza a las estructuras sociales por encima del individuo, le concede especial importancia a la socialización y a los procesos reproductivos de las pautas socioculturales, dando gran valor a la coacción y coerción social sobre el individuo, siendo la moral el elemento que permea todo el proceso educativo.

Vale destacar principalmente que para Durkheim, la socialización es de suma importancia, porque opera como el proceso de integración de los individuos a la sociedad y la adquisición por parte de estos de las competencias necesarias. El resultado final, es la dotación de un cierto modelo de individuo para una determinada sociedad en un espacio y tiempo definido. 
Otros de los conceptos claves para entender la obra durkheniana son "acción general", "acción colectiva", "solidaridad mecánica", "solidaridad orgánica" y "hecho social", pero debido a la amplitud de estos, no es posible desarrollarlos ampliamente.

Ahora bien, dentro de su obra se destacan ciertos estudios referentes a la educación como Educación y Sociología (1922/2009) donde analiza la educación desde los sistemas educativos que existieron y existen en cada sociedad, donde el sistema es único y múltiple a la vez, es decir, a todos los miembros de la sociedad le da ciertas pautas de uniformidad, pero también la educación varía según clases sociales, lugar de residencia, época, etc.

Una constante en su obra sobre educación, es la defensa del carácter social de la educación ante quienes privilegiaban su carácter individualista y psicológico, debido a que para el autor, la educación y el sistema educativo responden a necesidades eminentemente sociales. Además, dentro del sistema educativo reconoce el importante papel del Estado en establecer fines de índole social y el poder de la educación para tratar de alcanzarlos.

Por otro lado, reconoce la diferencia entre educación y pedagogía, "donde se desprende que la educación no es más que la materia de la pedagogía. Esta última estriba en una determinada forma de pensar respecto a los elementos de la educación" (Durkheim, 2009, p. 73). De esta forma la pedagogía como teoría práctica (negando su carácter de arte o ciencia) se apoya en las ciencias de la educación para su aplicación.

En cuanto a su relación con la sociología, Durkheim afirma que precisamente es esta ciencia la que ayuda a determinar los fines de la educación que se encuentran acordes con los fines de la sociedad y para lo cual es necesario la reproducción de las pautas socioculturales, donde cada norma e institución cumple con darle mantenimiento a la sociedad para que siga conservando su forma (Taberner, 2005).

Por su parte en La Educación Moral (1925/2002) realiza un análisis de la moral laica racionalista que aporta los elementos para el funcionamiento de la sociedad como un todo, donde la escuela apoya y resguarda el "tipo nacional francés" (imperante en la época en que escribe el texto), lejos de los dogmas y prácticas religiosas.

Para el autor, la educación moral sucede durante el proceso de la primera infancia dentro de la familia. Pero es principalmente en la segunda infancia que transcurre dentro de la escuela primaria y lejos de la familia, donde se constituyen las bases de la moral para la vida social fuera del círculo familiar.

De esta forma, el individuo en la infancia aprende las reglas generales, llamadas morales, como reglas preestablecidas para circunstancias particulares que "al agente moral le toca decidir cómo convenga particularizarla. Siempre hay un margen que se deja a su iniciativa, pero este margen es limitado" (Durkheim, 2002, p. 53).

Es aquí donde la sociedad a través de los agentes de socialización, como la escuela, impone, regula y establece el accionar de los individuos. Es decir, la moral (elemento externo al individuo) disciplina el accionar de cada uno de los individuos.

Dentro de su análisis, Durkheim propone que existen elementos de la moralidad, entre los cuales el primero es el espíritu de la disciplina, que establece conductas reguladas y fines determinados y define -al regular y contener- el horizonte de posibilidades del individuo en sociedad. 
El segundo elemento de la moralidad es la vinculación a los grupos sociales, que consiste en la integración del individuo en la sociedad a partir de un interés por vincularse, para lo cual debe abdicar a su naturaleza individual bajo la condición de formar parte de los grupos sociales. Pero en este punto salta la duda acerca de ¿qué espacio queda para el individuo? Es aquí donde surge el tercer elemento, la autonomía de la voluntad como elemento de la moralidad y parte de la antinomia entre las reglas morales exteriores a la voluntad y la autonomía individual.

Para Durkhreim (2002, pp. 105-106) bajo la influencia de Kant, esta antinomia se soluciona de la siguiente forma, "en la medida en que sólo nos mueva la razón, actuamos moralmente y, al mismo tiempo, actuamos con plena autonomía por que nos limitamos a seguir la ley de nuestra naturaleza razonable".

Por último, para inculcar en el estudiante los elementos de la moralidad, partiendo del espíritu de la disciplina (dominio personal y mesura de los deseos), se utilizan la disciplina escolar y los castigos. El autor analiza dos posiciones acerca del castigo: como forma de prevenir la falta de observancia de la regla (el castigo como amenaza e intimidación) y como forma de eliminar la falta (el castigo para reparar la infracción).

Ahora bien, la obra de Durkheim abarca diversos temas como La Evolución Pedagógica en Francia (1938/1980) (análisis de las determinantes históricas que dieron como resultado la pedagogía francesa de finales del siglo XIX y comienzos del XX); al igual que ponencias, ensayos o discursos, como El papel de las Universidades en la educación social del país (1900/2007) (análisis de las universidades de cara a la sociedad, los estudiantes y dentro del sistema educativo francés), La escuela del mañana (1915/2007) (visión a futuro del papel de la escuela en sociedad francesa post-primera guerra mundial), Debate sobre la Educación Sexual (1911/2007), entre otros.

Sin embargo, por la amplitud de su obra, solo se hará referencia al artículo sobre educación sexual. Este es una reproducción de una discusión entre Emile Durkheim y el doctor en medicina Jacques Doléris. En este caso Durkheim aborda lo que él llama el problema desde el punto de vista moral de la continencia de los jóvenes como un deber, ya que las uniones sexuales fuera del matrimonio provocan "conflictos sociales" de todo tipo (nacimiento de hijos fuera del matrimonio, por ejemplo).

Para Durkheim (2007, pp. 105-106) "la cuestión es más bien tratar de saber si se puede, y cómo, hacer comprender a los jóvenes que el estado del matrimonio se justifica desde el punto de vista legal, y que el comercio sexual fuera del matrimonio es inmoral".

La posición del autor es una crítica a la enseñanza de la higiene sexual (posición defendida por Doléris) debido a que deja de lado los aspectos sociales y morales vinculados con el acto sexual, y lo limita y define solo por su naturaleza física.

Por su parte, para Doléris la sexualidad debe ser vista lejos de los prejuicios, de los órdenes sociales y económicos, que ocultan la realidad del acto sexual. Pero coincide con Durkheim en cuanto a la prudencia, decencia y naturalidad con que el docente debe manejar el tema, sobre todo con las niñas.

En resumen, y como forma de recuperar los aspectos más importantes del pensamiento durkheniano, existe una moral que condiciona el accionar de los individuos, es decir, la forma de 
pensar, el comportamiento y la forma de sentir son impuestos desde el exterior del individuo. En el caso de la educación, los elementos de la moral deben ser inculcados en los jóvenes para lograr que las futuras generaciones cuenten con valores morales que a través de la coacción o coerción modelen el comportamiento de los individuos. Para esto la socialización se cubre de importancia como medio para el aprendizaje de las pautas que facilitan el introducirse en el mundo social, siendo estas pautas (junto con creencias, tendencias y prácticas) consideradas colectivamente.

Por último, bajo la influencia durkheniana (pero con marcadas diferencias y nuevos aportes), se puede ubicar los diversos trabajos de autores como Marcel Mauss (1872-1950), Claude Lévi-Strauss (1908-2009) y Pierre Bourdieu (1930-2002). Este último constituye un referente mundial acerca del estudio sociológico de la educación, lo cual quedó plasmado en su obra acerca del sistema educativo y la sociedad francesa del siglo XX (que se espera poder abordar en futuros ensayos o artículos).

También, para algunos estudiosos del tema, autores como Talcott Parsons (1902-1979) y Robert Merton (1910-2003) se ubican dentro de la influencia de esta tradición y forman parte de la llamada escuela estadounidense de sociología bajo el término "funcional estructuralismo". O el inglés Basil Bernstein (1924-2000), quien en una combinación de sociología y lingüística, se enfoca en el discurso pedagógico, el control social, las clases, la desventaja sociocultural, etc., dando como resultado otra vertiente para el estudio sociológico de la educación.

\subsubsection{El individualismo metodológico: Max Weber}

Esta corriente se relaciona con los aportes del filósofo, abogado y economista Max Weber (1864-1920), en lo que algunos autores llaman sociología comprensiva, de la interacción social, individualismo metodológico o accionismo.

En resumidas cuentas se puede decir que la perspectiva teórica weberiana se centra en la acción social y la interacción social (en este caso lo social es el resultado de esta interacción). Como señala en Economía y Sociedad (Weber, 1922/2008) la sociología es "una ciencia que pretende entender, interpretándola, la acción social para de esa manera explicarla causalmente en su desarrollo y efectos" (Weber, 2008, p. 5).

En el caso de acción, se define como "una conducta humana (bien consista en un hacer externo o interno, ya en un omitir o permitir) siempre que el sujeto o los sujetos de la acción enlacen a ella un sentido subjetivo" (Weber, 2008, p. 5).

Es decir, esta corriente teórica prioriza sobre el sentido y significado que los sujetos dan a sus acciones y decisiones individuales. Por eso, se concentra en el individuo y no en las estructuras sociales y le concede mayor importancia a la comprensión de la acción social (la cual tiene un sentido y significado) que a la coerción o coacción social (como en el caso de Durkheim).

Para este autor, la sociología debe encargarse de interpretar la acción a partir de la evidencia obtenida. Sin embargo, "la explicación interpretativa frente a la observación tiene ciertamente como precio el carácter esencialmente más hipotético y fragmentario de los resultados alcanzados por la interpretación. Pero es precisamente lo específico del conocimiento sociológico" (Weber, 2008, p. 13). 
Es en este punto donde la comprensión de la acción a través de la interpretación, que se pueda hacer de ella es la unidad de análisis de la sociología comprensiva, pero considerando que "la acción social no es idéntica a) ni es una acción homogénea de muchos, b) ni a la acción de alguien influido por conductas de otros" (Weber, 2008, p. 19).

De esta manera desde esta perspectiva lo social es definido por las interacciones dadas entre los sujetos y las consecuencias de las acciones individuales. Por su parte, la relación es una conducta plural que es recíprocamente referida y orientada por la reciprocidad.

Otros de los temas de interés y desarrollados por Weber son el poder, la comunidad, los tipos de dominación (lega, tradicional y carismática), el Estado (y su pretensión al monopolio legítimo de la violencia), etc.

Ahora bien, es importante acotar que a diferencia de Durkheim, Weber nunca dedicó un libro completo para estudiar el tema de la educación o el sistema educativo como tal. Sin embargo, aquí se recoge parte de su pensamiento asociado principalmente con las universidades de su tiempo.

En su obra Economía y Sociedad (2008), menciona la relación entre educación y profesión, ya que en la sociedad existen estructuras profesionales donde la articulación estamental y las formas de educación que se crean para clases de profesiones específicas (aristócratas, caballeros, clérigos, etc.) se involucran como cuadros administrativos encargados de realizar e imponer las ordenes y leyes dentro de cualquier forma de dominación.

No obstante, "el prestigio social basado en el disfrute de una determinada educación y de una determinada instrucción no es por sí mismo algo específico del burocratismo [...] en otras estructuras de dominación se basa en fundamentos esencialmente distintos" (Weber, 2008, p. 751).

Por otra parte, la educación suministra la base para una estratificación social de carácter estamental, dando lo necesario a los sujetos para ubicarse dentro de la sociedad en relación con otros estamentos y dentro del estamento al que pertenece en relación con sus pares.

En otras de sus obras, La ciencia como vocación (1919/ 2000) (que junto con La política como vocación, comúnmente se conocen como El político y el científico, aunque ningún texto de Weber lleva este título), describe y compara el sistema educativo universitario, a través del análisis de las funciones del assistant de las universidades americanas y del privatdozent de universidades alemanas, junto con otros aspectos acerca de la producción de conocimiento científico, el impartir lecciones en centros de enseñanza superior, el concurso por los puestos, etc.

Aunque previamente escribió Las universidades americanas y las alemanas (Weber, 1911/2007), donde se pueden observar ciertos elementos que dieron origen al texto en mención de 1919 y que permite reconocer un refinamiento de su posición sobre las universidades alemanas y la carrera docente.

Ahora prosiguiendo con La ciencia como vocación, a partir de su experiencia como docente, es tajante al denotar, primero, que "sería injusto culpar a la inferioridad personal del Ministerio de las Facultades del hecho indudable de que existan muchos mediocres que ocupan puestos importantes en las universidades" (Weber, 2000, p. 187).

Segundo, "todo joven que se crea llamado a la profesión académica debe tener conciencia clara de que la tarea que le aguarda tiene una doble vertiente. No le bastará con estar 
cualificado como sabio, sino que ha de estarlo también como profesor, y estas dos cualidades no se implican recíprocamente ni mucho menos" (Weber, 2000, p. 189).

Tercero, el "éxito" de un curso no es algo cuantificable, debido a que todo depende de la asistencia de los estudiantes y esta a su vez depende de circunstancias externas como "el temperamento del profesor o su timbre de voz" (Weber, 2000, p. 190). Por eso, Weber desconfiaba de los cursos con salones llenos de estudiantes, debido a que son engañosos en cuanto a la calidad de la enseñanza impartida.

Cuarto, para Weber las opiniones políticas de los profesores no deben marcar a los estudiantes, aunque resulte imposible prescindir de toda simpatía política de carácter subjetivo por parte del profesor hacia ciertos temas. Esto con el fin de valorar el lugar del conocimiento científico y no introducir en los salones de clase los juicios políticos valorativos del docente.

Por otro lado, a través de ensayos o intervenciones en congresos, Weber realizó sus otros aportes al análisis de la educación en la Alemania de finales del siglo XIX y comienzos del XX.

Así por ejemplo, en La pretendida libertad intelectual en las universidades alemanas (1908/2007) Weber profundiza en la libertad intelectual de los profesores de educación superior, siendo esta atentada por el clericalismo y otros elementos, al igual que el interés del autor por dilucidar sobre los alcances de la libertad intelectual, ya que para él "esta pretendida libertad depende, en primer lugar, de la adhesión a opiniones bien vistas en la Corte y en los salones, y, en segundo lugar, de que vaya acompañada de la expresión de un mínimo o de una apariencia de conformismo religioso" (Weber, 2007, p. 53).

Dos años más tarde, en La libertad intelectual en las universidades (Weber, 1909/2007, p. 56) vuelve a tocar este tema en relación a "la libertad de la ciencia, de la investigación y de la enseñanza" y el papel de la iglesia y el Estado como condicionantes de esta libertad y de la permanencia o no de un profesor en su puesto.

De esta manera es claro que en el caso de Weber, no existe un interés particular por investigar a fondo el tema de la educación como si sucedió en el caso de Durkheim. No obstante, se puede reconocer que para Weber la educación se encuentra asociada con la división social en clases y estamentos.

Bajo la influencia weberiana, se pueden clasificar los trabajos en dos ramas distintas. Por un lado, Jean-Claude Passeron (1930-?) y la teoría de la reproducción desarrollada junto con Pierre Bourdieu, por otro lado, Raymond Boudon (1934-?), quien para Van Haecht (1999) representa de mejor manera el individualismo metodológico.

De forma paralela al accionismo, pero influenciada por esta corriente, surgió durante la segunda mitad del siglo XX la sociología de la acción o accionalismo, cuyos puntos de análisis han sido los movimientos sociales, los conflictos sociales en busca de orientar el sentido de la sociedad y la transformación del sujeto en actor durante este proceso.

El principal representante de esta corriente es Alain Touraine (1925-?), que aunque no escribió o investigó sobre la educación exclusivamente, es importante destacar su presencia y ciertas referencias que hizo acerca de la educación en el momento actual.

Para Touraine (2009) la sociedad, y específicamente la francesa, se enfrenta a una falta de reconocimiento de las características individuales de los estudiantes por parte de la 
escuela, la resistencia a la integración de sectores caracterizados por su pluriculturalidad, un falso igualitarismo en el sistema educativo, el papel de la escuela como transmisora de desigualdades sociales, entre otros.

Como discípulo de este último, se menciona a François Dubet (1946-?) quien en varios estudios se ha enfocado en el tema de la educación, en particular sobre la realidad acerca del mérito y el tema de las oportunidades dentro de los centros educativos, en lo que ha definido como "la igualdad meritocrática de oportunidades" (Dubet, 2006, p. 19).

Ahora bien, como se puede observar dentro del individualismo metodológico existen una diversidad de autores que siguen lo planteado por Weber pero no de manera estricta, sino que utilizan las premisas de Weber (y de otros autores) dentro de sus análisis de la realidad social. Es por este motivo que no se puede hablar específicamente de "una escuela weberiana".

\subsubsection{La perspectiva marxista}

En general, la obra de Karl Marx (1818-1883), y la desarrollada en conjunto con Friedrich Engels (1820-1895), ha sido una de las perspectivas teóricas que mayor polémica y debate ha causado, ya sea a favor o en contra de lo planteado originalmente por Marx.

No obstante, la cantidad de seguidores de la obra de este pensador se ha extendido en todas las ciencias sociales y ha evolucionado hasta producir diversas posturas (como los estructuralistas marxistas y los neomarxistas), dando paso a numerosas tendencias basadas en su obra.

En general, los planteamientos de esta perspectiva se pueden resumir de la siguiente forma: Aunque la sociedad es vista como un "todo", esta se encuentra divida en clases sociales con intereses antagónicos. En El Manifiesto del Partido Comunista (1847/1997) se postula que la historia de las sociedades humanas es el resultado de la lucha entre clases sociales.

Este conflicto entre clases (por el que algunos llaman a este enfoque como Teoría del Conflicto), da paso a relaciones entre obreros (quienes forman la fuerza de producción que la intercambian por un salario) y burgueses (quienes controlan los medios de producción: talleres, fábricas, etc.).

Para Marx, cada sociedad viene acompañada de su propio modo de producción: "por eso para comprender el «modo de producción» social de la existencia hay que considerar las técnicas de producción existentes (fuerzas productivas), y la propiedad de los medios de producción + las condiciones de trabajo (relaciones sociales de producción)". (Taberner, 2005, p. 25).

Esto da como resultado la existencia de dos clases sociales de mayor relevancia (obreros y burgueses) que se encuentran enfrentadas. Pero además reconoce la existencia de capas medias, lo cual genera una dinámica social constante entre clases sociales.

De esta manera, conceptos como «modo de producción» y «clases sociales», permiten afirmar que existe un interés de esta perspectiva teórica por aquellos elementos económicos y políticos que median entre la explotación de la clase obrera y la producción material de la sociedad.

Por otro lado, dentro de este enfoque se representa a la sociedad como un edificio. En este caso existen dos elementos a considerar como lo son la estructura (o infraestructura, donde se encuentran las fuerzas productivas y las relaciones de producción que proveen de 
la base material a la sociedad) y la superestructura (donde se ubica lo ideológico por medio de leyes, política, lo jurídico, etc., e instituciones como el Estado, la escuela, la iglesia, las manifestaciones artísticas, etc.).

De este modo, la superestructura se "apoya en la base" y lo que ocurre en la superestructura se ve determinado por lo que pueda suceder en la infraestructura. Al respecto, Althusser (1970/ 2008, p. 17) ve en esto una "determinación en última instancia de lo que ocurre en los "pisos" (de la superestructura) por lo que ocurra en la base económica."

Aun así, el mismo Marx, como lo destaca Althusser, reconoce que lo que sucede entre superestructura y estructura es pensado bajo dos formas: la autonomía relativa de la superestructura y la reacción de la superestructura sobre la base.

Ahora bien, en relación con el antagonismo de clases, esto sólo puede resolverse a través de la dictadura del proletariado, es decir, la toma del poder político por parte del proletariado y la supresión de la propiedad privada burguesa.

La solución que se plantea es socializar los medios de producción y con esto hacer tambalear la estructura social, lo cual significaría un cambio radical aboliendo la explotación y la propiedad privada, eliminando las clases sociales a favor de los intereses de la colectividad.

Por supuesto, esto último representa parte del "Marx político" y no tanto del "Marx científico", debido a que en ocasiones el autor intercalaba en su obra ciertos aspectos de su visión política de la situación de Europa del siglo XIX, durante la segunda Revolución Industrial. Es en este punto donde la revolución de la sociedad representa un cambio profundo, pero necesario desde la perspectiva marxista, para alterar la situación de la clase proletaria.

Por otro lado, aunque el tema de la educación no fue el centro de sus trabajos más representativos, el autor desarrolló algunos aspectos referentes a la educación, como en $E l$ Capital (Tomo I), Trabajo de los niños y escolarización (1867/ 2007) y en ciertos escritos como Exámenes y Burocracia (1843/2007), tomado de La crítica de la filosofía del derecho de Hegel, entre otros.

En el primero de ellos, señala la situación de explotación que sufren mujeres y niños en las fábricas y empresas. Ante tal situación el Parlamento inglés estableció como obligatoria la enseñanza hasta los 14 años, pero dicha ley no se cumplió. Lo anterior, junto con las condiciones de las escuelas son, para el autor, producto de la desprotección del Estado y la explotación burguesa.

Sobre Exámenes y Burocracia, señala que la burocracia es una jerarquía del saber entre los distintos estratos de la pirámide del poder burocrático, lo cual no está al alcance de todos. En este punto, los exámenes se convierten en la entrada a los privilegios del círculo de la burocracia, restringidos sólo para quienes aprueben los exámenes.

Por lo tanto, aunque en las obras de Marx el tema de la escuela o la educación no fue algo ampliamente desarrollado, al conocer las proposiciones marxistas se puede reconocer que la educación y la escuela son producto de la superestructura, donde el factor ideológico de la sociedad capitalista busca la reproducción de las condiciones de producción y con esto mantener a la burguesía en el poder.

Finalmente, es importante señalar que durante el siglo XX el tema de la educación y el sistema educativo son expuestos de forma más explícita en la obra de autores como Louis 
Althusser (1918-1990), Antonio Gramsci (1891-1937), Theodor Adorno (1903-1969), Jean-Michel Berthelot (1945-2006), entre otros, desde la perspectiva marxista. No obstante, no se detallará en este trabajo los aportes de estos autores en este campo, dado que esto es tema de futuros ensayos o artículos debido a la diversidad y amplitud de estos enfoques.

\subsubsection{Los aportes e influencia de Simmel}

De todos los clásicos, es quizá Georg Simmel (1858-1918) el único que no goza de un amplio prestigio en Latinoamérica como los anteriores (Durkheim, Weber, Marx), debido, tal vez, al desconocimiento de su obra o la subvaloración de la misma.

Recientemente en español ha sido publicada la obra Pedagogía Escolar (Simmel, 1921/2008), la cual recoge sus lecciones sobre pedagogía (impartidas en el Jardín de Botánica de la Universidad de Estrasburgo, único lugar que quedó disponible al ser ocupadas las aulas como hospital militar durante la Primera Guerra Mundial), escritas por Simmel pero editadas por Karl Hauter.

Watier (2005) señala que este enfoque teórico se caracteriza por considerar a la sociología como una ciencia ecléctica, que toma de otras ciencias (Historia, Antropología, etc.) materiales para elaborar una síntesis con base en una nueva mirada (mirada sociológica) sobre los hechos, por eso las obras de Simmel abarcan un amplio espectro de ciencias sociales y la filosofía.

Esta mirada parte de la premisa de tomar cierta distancia para registrar los hechos y la experiencia que sucede en la realidad social. Pero valora que estos hechos puedan tomar el lugar de conocimientos como una construcción, producto de una interpretación y un modelado por parte de los individuos que se encuentran inmersos en numerosas ligaduras sociales.

Por otro lado, para Simmel la sociedad es posible gracias a la conciencia de ser socializado, de quienes integran la sociedad. Esta se da bajo una reciprocidad, pero no se da en forma total y mucho menos de la forma idealizada que denotan algunos autores (Watier, 2005), dando paso a una correlación entre el individuo y la aparición de colectivos, de los cuales forma parte.

Por eso, para este autor la aparición de grupos y las formas de ligaduras y relaciones asociativas entre sus integrantes se cubren de especial importancia, ya que dan paso a la formación de colectivos.

Otro aspecto importante del análisis de Simmel son las formas de socialización, las cuales cargan con un contenido (intereses, pulsiones, disposiciones, sentimientos, etc.), en las cuales para conseguirlos cada individuo va a materializarlos a través de diversas formas de socializarse.

Por eso, si bien para Simmel son importantes las instituciones sociales, es más importante el estudio de las asociaciones como parte de las relaciones de unos con otros, dinámica en la que la socialización se convierte en un vínculo entre individuos y genera diversos grados de cohesión entre los mismos.

Estas formas de asociación son más o menos conscientes. Así el contenido de una socialización puede generar asociaciones y estas a su vez genera la aparición de colectivos, de manera que, entre más elevado sea el grado de conciencia de aquello que los une, más alto será valorizada esa ligadura que los une. 
Para aclarar lo anterior es necesario compararlo con lo indicado por Durkheim, el otro autor que le da énfasis al tema de la socialización, de forma que "un durkheimiano diría que el número y la intensidad de las relaciones contribuyen a la densidad moral de la sociedad, y Simmel añade a ello claramente una posibilidad de expansión personal e individual" (Watier, 2005, p. 25).

En el campo de la educación, Simmel (2008) plantea que a la pedagogía comúnmente no se le reconoce como ciencia, pero para él la pedagogía es un arte dentro de la praxis pedagógica de quien enseña, el cual necesita de un saber. Por eso aclara que

(...) no sólo de uno [de un saber] en un sentido técnico, que consistiría en el aprendizaje práctico de pequeños ejercicios manuales, sino un saber sobre el material, sobre la extensión y límites de las tareas, sobre el campo global de principios y máximas que está entre la última forma, decidida sólo a través del talento o del genio, y lo técnicoartesanal, y que hace a lo último adaptable a lo primero, y que traslada a éste hacia aquél (Simmel, 2008, pp. 11-12).

En el libro, Simmel trata de exponer su visión sobre el "arte de enseñar" y la educación, con base en la relación educación-enseñanza, los aspectos generales de la enseñanza y su aplicación en materias específicas como matemáticas, idiomas, historia, etc.

Para Simmel la educación es un proceso para la vida y no sólo para la sociedad (como en el caso de Durkheim). Por tal motivo no visualiza al estudiante como un agente pasivo, sino todo lo contrario, lo visualiza como un agente activo que al igual que el docente aspiran a alcanzar el conocimiento.

Por eso, para Vernik (2008) dentro del arte de enseñar de Simmel, se conjuga la fragmentación de su tiempo (una modernidad que prioriza lo fragmentario -fin de las verdades absolutas y la fe como respuesta del mundo- y relativo), la vivencia (sobre todo pedagógica, donde se combina la comprensión de docente-estudiantes) y la libertad (como forma de individualidad, para resolver problemas, y como formación y autoformación).

En síntesis, Vernik (2008) señala aspectos claves del pensamiento pedagógico de Simmel, a saber: concebir la clase como una totalidad (cada clase es una unidad pero que debe estar conectada con la siguiente), suscitar la atención de los estudiantes (el éxito de una clase depende del grado de atención estudiantil logrado por el docente), transmitir el interés propio (despertar en el estudiantado el interés por el objeto de estudio), contra la fragmentación (articular los contenidos con el fin de la formación integral de la persona), articular los contenidos (plantear de forma sistemática y articulada donde se conjuguen las diferentes disciplinas), a favor de las personalidades (nombrar a los alumnos y reconocer sus particularidades), contra la rutina (por su afectación a la enseñanza).

Otro punto clave de la obra de Simmel es su apreciación sobre el proceso de enseñanza y aprendizaje. El autor considera que este no debe centrarse exclusivamente en el aprendizaje del alumno de los contenidos, sino que además en el aprendizaje de "la forma del desarrollo en lo espiritual" (Simmel, 2008, p. 16).

Por último, para Simmel (2008) los contenidos deben presentarse bajo un método genético de perspectiva histórica y sistemática: de lo más simple a lo más complejo, donde el 
docente asume los contenidos como materia prima de un proceso de rendimiento individual y vivo, debido a que Simmel visualiza al estudiante como un agente activo del proceso de enseñanza y aprendizaje.

Ahora bien, en este punto se espera que el lector y la lectora tengan una perspectiva más amplia sobre la sociología como ciencia social y sobre sus distintas tradiciones teóricas. Este repaso por las perspectivas teóricas más influyentes (Durkheim, Weber, Marx, Simmel) se hizo necesario justamente para responder a las preguntas iniciales: ¿qué es? Y ¿para qué sirve la sociología? En este caso interesaba principalmente que dichas respuestas subrayaran los aportes de dicha ciencia al campo específico de la educación.

Conviene señalar que en esa misma línea, algunos autores importantes han continuado estas corrientes teóricas detalladas antes en épocas más recientes y han profundizado en este tema desde un enfoque claramente sociológico y otros han realizado un aporte propio. Entre estas se encuentra la obra de Karl Mannheim (1893-1947), que desde de la sociología del conocimiento realizó ciertas observaciones sobre la educación en su paso por Inglaterra, que fue editado de forma póstuma (Kupiec, 2008) e inspiró a muchos investigadores ingleses como Ottaway (1965), quien reconoce la importancia de la obra de Mannheim en el desarrollo de la sociología de la educación inglesa y del interés por el pensamiento sociológico por parte de la generación de estudiantes de docencia en ese momento y posteriormente.

No obstante, continuar con los abordajes teóricos sería extender la discusión sin llegar a cumplir el objetivo de este trabajo (reflexionar sobre el objeto de la sociología de la educación). En futuros ensayos o artículos se retomaran con detalle estos aportes de autores más recientes.

En este caso interesa ahora reflexionar acerca del "segundo elemento de la ecuación", en otras palabras ¿qué es la educación? Para pasar posteriormente a enfocarse en el objeto de estudio de la llamada sociología de la educación, debido a que en ocasiones parece no haber claridad ni consenso entre los autores clásicos.

\subsection{Una definición de educación}

Lo anterior lleva a formularse preguntas como: ¿qué se entiende por educación? ¿Cómo se da la educación? ¿Quiénes intervienen y de qué forma en la educación? ¿Qué aspectos sociales, económicos, culturales, etc., se encuentran involucrados con la educación?

Para poder contestar algunas de estas preguntas y estudiar la educación como objeto de estudio, las y los educadores cuentan con la pedagogía y esta a su vez cuenta con las llamadas ciencias de la educación (donde la sociología es tan solo una de ellas), debido a la complejidad del fenómeno educativo.

Al respecto Gómez y Domínguez (2001, p. 23) tratan de definir educación y señalan la pluralidad de definiciones existentes.

(...) de la veintena de definiciones de educación que podemos encontrar en los manuales de pedagogía citados en este tema, nos daríamos cuenta de que, salvadas las distintas perspectivas, hay un substrato común a todas ellas y es que la educación es un proceso de integración del individuo en la sociedad. 
Si bien tal afirmación es acertada, cometen varios errores muy comunes a la hora de brindar una definición sobre la educación, que desde el enfoque aquí expuesto, no se adaptan a los tiempos actuales, ya sea porque de forma similar como señala Horkheimer (2000, p. 23) para el caso de la teoría "se ha observado mal, o bien hay algún error en los principios teóricos".

Para aclarar mejor lo anterior, se presenta la definición de los autores y se señala lo que se considera, son errores en su definición:

Habrá siempre un educando y un educador [Error 1] (...) El educado es el niño (...) el educador es un adulto [Error 2] (...) el adulto sirve y dirige al niño para conducirlo a aquellos comportamientos que, en su sociedad concreta, sean considerados como deseables [Error 3] (...)El adulto que está integrado en la sociedad, tiene la ciencia y la experiencia social y lleva de la mano -ese es el significado de la palabra pedagogo- a un niño, sabiendo el camino por el que lo tiene que llevar [Error 4] (Gómez y Domínguez, 2001, p. 23).

Primero, en la época actual la relación primigenia "educando y educador" se ha visto modificada por los adelantos tecnológicos y aunque dicha situación se presenta con mucha frecuencia, no es una característica determinante para reconocer a la educación como tal.

Actualmente el educando se puede relacionar con una computadora y bases de datos que le brinden la información necesaria, sin mediar en la ecuación el educador (cursos no presenciales, cursos virtuales, etc.). De igual forma puede ser evaluado a través de un sistema automatizado y conocer su nota al final de la aplicación de la prueba.

Segundo, la relación niño-adulto tampoco es una característica propia de la educación, debido a que existe la educación adulto-adulto. Acerca de esto, señala Terrot (Le Bouëdec, 2000) que se pueden reconocer tres fases dentro de la educación para adultos como parte de su evolución:

Fase 1. Alfabetización, para dotar a la población adulta analfabeta de las herramientas necesarias para desempeñar los oficios de la época.

Fase 2. Recuperación escolar, ante las insuficiencias del sistema educativo en aquellas personas que no pudieron tener una permanencia prolongada en él. Es en este momento histórico (primera mitad del siglo XX) que en Francia se comienza a hablar de educación profesional, educación obrera y educación popular, siendo los tres componentes de la educación para adultos en gran parte del siglo XX.

Fase 3. La formación continua, producto de los cambios tecnológicos y económicos, se plantea la necesidad de contar con la formación como una constante dentro de las empresas para "actualizar" al personal.

Por lo anterior, ante tan clara evidencia es absurdo pensar que la relación educador-educando sólo se pueda dar entre un adulto y un niño. Esto de forma implícita deja observar que algunos autores restringen a la educación a las aulas y a los centros educativos que forman parte del sistema de educación formal, dejando de lado la educación no formal como parte del aprendizaje de ciertos sectores de la sociedad.

Tercero, desde la definición de Gómez y Domínguez (2001) el educador es el único mediador en la socialización de la persona menor de edad, es decir, exclusivamente es el responsable de la adquisición por parte del educando de "los comportamientos deseables". 
En este punto se debe ser categórico al decir que cualquier centro educativo es un escenario social donde la interacción entre los posibles y distintos sujetos involucrados en el proceso de enseñanza y aprendizaje, aportan a la creación de una red amplia de relaciones e interacciones dentro de las cuales la socialización es un proceso y no responsabilidad de una sola persona.

En otras palabras, personal administrativo-docentes-madres y padres-estudiantescomunidad-Estado, configuran el escenario educativo donde lo institucional, lo intersubjetivo y lo subjetivo intervienen en la formación del educando.

Cuarto, que el adulto tenga "las llaves" para entrar a la sociedad, el conocimiento y la experiencia social, es volver a las visiones jerarquizadas y jerarquizantes, estructuradas y estructurantes del docente como un ser "omnisapiente e infalible" y del estudiante como un “cascarón hueco y vacío de conocimiento", el cual debe el docente llenar con su "sabiduría".

Sin duda la pedagogía ha evolucionado a visiones donde el educador es un facilitador, orientador y mediador entre el conocimiento y el estudiante. La labor del docente es de facilitar el proceso de aprendizaje a través de una enseñanza que al emplear medios, técnicas e instrumentos novedosos, permitan una cierta autonomía del educando para definir su proceso de aprendizaje.

A esto se debe sumar que la escuela es un espacio caracterizado por la diversidad en sus diferentes sectores poblacionales. Un ejemplo de esto es el caso de la población estudiantil, que durante mucho tiempo se le consideró como un grupo homogéneo. No obstante, la visión ha cambiado, ya que ahora se le define como un grupo heterogéneo debido al origen familiar, sexo, edad, condiciones socioeconómicas, lugar de residencia, capacidades sociocognitivas, etc.

Además, la educación se da en múltiples escenarios y su realidad es una pluralidad de situaciones y bajo circunstancias diversas.

De forma indirecta, esto vendría, por un lado, a confirmar el carácter cambiante de la educación por lo cual es importante observarla desde una perspectiva histórica. Por otro lado, permitiría reconocer que actualmente es necesario convertir a los centros educativos en lugares de integración, donde la pedagogía englobe temas como la interculturalidad, diversidad, derechos humanos, prevención de la violencia, educación para la paz, etc.

Por eso, así como los centros educativos han cambiado, también ha cambiado la visión acerca de la población estudiantil, reconociendo la diversidad y las necesidades individuales. Esto queda claro con el caso de la población con necesidades educativas especiales y los enfoques que ha adoptado la educación especial, respecto a lo cual Gardou (2000, p. 255) señala que ha evolucionado "desde la internación en los asilos, pasando por la creación de clases especiales, hasta el desarrollo de los centros especializados y los recientes dispositivos con miras a la integración."

Por otro lado, como indica Díaz (2009), al revisar la historia de la educación se puede notar que esta es un proceso cambiante, producto de las transformaciones de las sociedades. Específicamente durante la segunda mitad del siglo XX se abrió la "oferta de pedagogías" a las diversas corrientes pedagógicas del momento: enseñanza programada y tecnología educativa, psicología cognitiva (Bruner, Ausubel), la pedagogía liberadora (Freire), constructivismo (Vitgosky, Piaget), etc., cada una con diferente "intencionalidad" acerca del modelo de sociedad, educando y ciudadanía que desean construir. 
De esta forma, se podría definir la educación como un proceso cambiante que se desarrolla durante todo el ciclo vital (pero con especial énfasis en la niñez, adolescencia y juventud) para poner al alcance de las personas valores, conocimientos, desarrollo de destrezas, pautas socioculturales, etc., que permitan una mejor inserción del individuo en la sociedad y un desarrollo personal de las capacidades (físicas e intelectuales) y actitudes de cada quien, en busca de un desarrollo pleno del bienestar personal y social del individuo.

Este proceso se puede dar tanto dentro como fuera del sistema educativo formal y puede darse como forma de interacción (en la relación educando-educador) como de forma individual a través del desarrollo de técnicas de estudio individual y autodidacta, amparadas por el desarrollo tecnológico.

Ahora bien, ya que se definió «sociología» y «educación» es preciso ahondar en la relación de estas dos, de cara a definir el objeto de estudio de la sociología de la educación.

\subsection{El objeto de estudio de la Sociología de la Educación}

Al hablar del objeto de estudio de la sociología de la educación es necesario reconocer que se debe hacer ciertas observaciones al respecto, porque en ocasiones al leer lo que se ha escrito sobre el tema (por ejemplo, en el caso de los autores clásicos antes mencionados) parece detectarse una falta de claridad sobre el objeto de estudio de esta rama de la sociología.

Para ejemplificar lo antes dicho, de forma resumida se puede notar que para Durkheim la educación es el curriculum, tanto oficial como oculto, debido a que se enfoca tanto en los contenidos como en los valores morales. En cambio para Weber la educación interviene en la sociedad por medio de los estamentos. Por otro lado, Weber también se enfoca en el tema del poder detrás del nombramiento de profesores universitarios y sostiene que inevitablemente las influencias políticas condicionan el avance o estancamiento de algunos.

En cambio, a Marx le interesa destacar el factor ideológico de la educación detrás de la dominación y la reproducción de las condiciones de producción y la marginalidad que sufre la clase proletaria respecto a las condiciones en las que se desarrolla su educación.

Simmel por su parte centra su atención en el proceso de enseñanza y aprendizaje, el papel del docente, la metodología en las lecciones, etc., y en el acontecer de la lección y la educación.

No obstante, para comenzar con el análisis del objeto de estudio de la sociología de la educación, se puede mencionar lo apuntado por Van Haecht (1999, p. 9) al inicio de su libro:

Arriesgar una introducción a la sociología de la educación en lengua francesa no es una empresa simple cuando se sabe que detrás de la traducción de una fórmula anglosajona (Sociology of Education) se esconde una (o, mejor dicho, unas) sociología(s) de la escuela.

Porque, en definitiva, ¿no nos sentimos hoy acaso divididos entre la sensación de que ya se ha dicho todo sobre este tema y la impresión de que son muchas las incógnitas que todavía sobrevuelan los desafíos sociales que allí se despliegan? (original en cursiva).

De lo anterior se puede reconocer que, lo que comenzó siendo un campo de investigación definido por investigadores ingleses y que se delimitaba a la escuela, ha evolucionado pero no 
sin evitar "incógnitas" o una pluralidad de enfoques que dan como resultado una constelación de posibles objetos de estudio para la sociología de la educación.

Ante esto, de forma propositiva se podría sugerir que la sociología de la educación se ocupa de un "macrotema" porque "la materia es, en efecto, inmensa y poco definida" (Janne, 1980, p. 13), donde se podría reconocer múltiples definiciones sobre el objeto de estudio desde diversas perspectivas.

De esta forma, como aporte sobre el tema, luego del repaso de todo lo antes mencionado, es preciso "catalogar" el horizonte de todo lo observable en materia de sociología de la educación, porque una clasificación brinda una mayor claridad acerca del tema. En este caso, el estudio sociológico de la educación es un campo amplio que se puede seccionar y clasificar de la siguiente forma:

- La "visión tradicional" de la sociología de la educación: El estudio de la relación sociedad-educación. No obstante, a esto se podría adicionar lo que Gómez y Domínguez (2001) señalan sobre la relación recíproca, porque además hay una interacción de la educación para con la sociedad, donde la educación se hace visible de múltiples formas (ejemplo, como un proceso de aculturación para el estudiante).

- Otras visiones: De forma propositiva se clasifican todas estas visiones en tres tipos: sociología educativa, sociología del sistema educativo y sociología educacional.

De lo anterior se puede decir que la sociología se puede ocupar tanto de los factores sociales que puedan tener una consecuencia o relación con la educación y viceversa, como de las instituciones educativas (en general y no solo de las escuelas) en calidad de institución social y escenario social donde ocurre la interacción social de numerosos individuos (docentes, estudiantes, madres, padres, etc.).

Profundizando en la clasificación de las otras visiones y como parte de la propuesta para abarcar el objeto de estudio de esta rama de la sociología, es preciso diferenciar entre:

- Sociología educativa: El estudio de la parte programática de la educación, en relación con la selección y planificación de los contenidos, actitudes, comportamientos deseables en las educandas (os), valores, actitudes, etc., los cuales son socialmente determinados, legitimados e incluidos por el Estado en sus programas y políticas educativas. En resumidas cuentas la labor de la educación (desarrollo de capacidades y posibilidades) y la formación (ejercicio de la aptitud adquirida) (Avanzini en Le Bouëdec, 2000).

El factor ideológico detrás de las propuestas educativas sería un aspecto por abarcar dentro de la línea investigativa de la sociología educativa, donde se proyecta la construcción de cierto tipo de ciudadanía con base en los contenidos seleccionados.

- Sociología del sistema educativo: Se estaría, por un lado, ante el sistema como institución, dando como resultado lo que Gras (1980) define como sociología de las instituciones educativas. En este caso se relaciona el sistema educativo con la sociedad. Pero esto se puede ampliar, al entender el sistema también como un espacio 
laboral del personal docente. Además, entrarían dentro de la sociología del sistema educativo el estudio del sistema de admisión, de promoción, escogencia de carrera, etc., de cualquier centro educativo donde se presente una selección de estudiantes de entre una población mayor.

Como ejemplo se menciona la sociología de la educación enfocada en el estudio del sistema educativo. Esta no sólo debería preocuparse por estudiar las condiciones en las que llegan los estudiantes a presentar el examen de admisión de una universidad, sino también los factores que puedan incidir en la escogencia de carrera (al respecto es revelador el estudio de Bourdieu y Passeron (2006) en Los Herederos), e incluso observar los resultados al final del proceso, es decir, con base en el número de graduados, analizar sus posibilidades de ingreso al mercado laboral con la preparación recibida.

Por otro lado, desde la sociología del sistema educativo se visualiza al sistema como escenario social, donde se producen interacciones sociales con el contexto comunitario y entre estudiantes, docentes, administrativos, etc.

- La sociología educacional: "que se interesa por el desarrollo de los conocimientos de carácter sociológico y psicosociológico que tienen relación directa o conexiones lógicas con los problemas de la práctica educativa" (Jensen, 1971, p. 11), es decir, enfocada en la interacción y problemática que acontece en la práctica educativa, siendo esta una acción de índole social.

La propuesta de clasificación anterior responde a la convicción de que existe una relación recíproca entre sistema y educación, pero esta relación se propone hacerse más amplia para incluir la sociedad, el Estado y el mercado, ya que la educación viene acompañada del sistema que le permite su aplicación, y estos responden a la influencia de la sociedad y viceversa (ver figura 1).

\begin{tabular}{|c|c|c|c|c|}
\hline Sociedad & Educación & Estado & $\begin{array}{c}\text { Sistema } \\
\text { educativo }\end{array}$ & Mercado \\
\hline $\begin{array}{l}\text { La sociedad } \\
\text { define valores, } \\
\text { comportamientos, } \\
\text { etc., incorporado en la } \\
\text { educación }\end{array}$ & & $\begin{array}{l}\text { Define la educación y } \\
\text { la forma del sistema } \\
\text { (curriculum, promoción, } \\
\text { política educativa, } \\
\text { reclutamiento personal } \\
\text { docente, etc) }\end{array}$ & & $\begin{array}{c}\text { El estilo de } \\
\text { desarrollo influye } \\
\text { en el mercado } \\
\text { laboral y generación } \\
\text { de recursos para } \\
\text { presupuesto nacional }\end{array}$ \\
\hline $\begin{array}{l}\text { El contexto comunal } \\
\text { incide en la población } \\
\text { estudiantil y en el centro } \\
\text { educativo (clases sociales, }\end{array}$ & $\begin{array}{c}\text { Incide en la sociedad } \\
\text { a través de la } \\
\text { socialización (un tipo } \\
\text { de ciudadanía a una } \\
\text { determinada sociedad) }\end{array}$ & $\begin{array}{l}\text { El Estado propara mano } \\
\text { de obra para el mercado } \\
\text { laboral privado y público }\end{array}$ & $\begin{array}{l}--------- \\
- \\
\text { El sistema incorpora al } \\
\text { estudiantado al mundo } \\
\text { laboral o educación } \\
\quad \text { superior } \\
\end{array}$ & \\
\hline & -------- & ---------- & $\longrightarrow$ & \\
\hline
\end{tabular}

Figura 1. Interacciones entre sociedad-educación-Estado- sistema educativo-mercado.

Nota: Elaboración propia. 
Esto lleva a plantear aspectos medulares acerca de la investigación sociológica en educación y lo que es "sociológicamente apropiado", por decirlo de alguna forma, de investigar en el campo de la educación. Para esto, dentro del esquema anterior solo pretende denotar algunas de las múltiples interacciones en las que la educación y el sistema educativo se ven envueltas. Por ejemplo, donde la sociedad aporta valores, comportamientos, etc., socialmente considerados como aceptables y que son incorporados en la educación.

En el caso de la incidencia del contexto comunal en el sistema educativo, se hace referencia a las desigualdades sociales, exclusión social y otros aspectos que inciden en el centro educativo y en su población estudiantil.

Por su parte, la educación también tiene una influencia en la sociedad. Por ejemplo, para Ottaway (1965) existe una relación entre educación y sociedad a través de la cultura, pero que también está destinada a producir un determinado tipo de ciudadana y ciudadano. Por eso para este autor, la sociología en relación con la educación debe estudiar su influencia social y su papel en el desarrollo del individuo.

En el caso del Estado, este juega un papel importante. Aunque algunos autores hablan de una nueva gestión de la educación (Whitty, Power y Halpin, 1999) con una cierta visión clientelar y un cambio en la toma de decisiones, sigue siendo, sin duda, el Estado quien determina la propuesta programática para las instituciones de educación y la estructura del sistema educativo.

En última instancia, el Estado busca construir a través de la educación un tipo de ciudadanía y garantizarse mano de obra para cubrir las necesidades del mercado laboral. Ejemplo, dentro del sistema de educación formal, los colegios vocacionales buscan generar estudiantes de diversas disciplinas para cubrir la demanda de mano de obra técnica.

No obstante, el presupuesto para educación, depende de las entradas de la hacienda pública provistas por las actividades económicas y el sistema tributario del Estado. En resumen, el presupuesto del Estado depende de la situación económica del país y esto a su vez repercute en las posibilidades de inversión en el sistema educativo.

Por otro lado, es prioritario preguntarse ¿qué hace a una investigación ser considerada como un estudio en sociología de la educación? Al pensar la respuesta, surgen más preguntas: ¿el mero hecho de que la realizó alguien graduado en sociología? ¿Qué entrelaza los fenómenos educativos con los demás fenómenos de la sociedad? ¿Qué sigue los pasos del método científico? ¿La sola mención de palabras como "educación”, "escuela” y "sociedad” hace a una investigación sociológica pertinente al campo de la sociología de la educación?

Como se señaló, aunque la sociología cuenta con una funcionalidad clara en cuanto a la descripción, interpretación y explicación de la realidad social como parte de su visión crítica (Lahire, 2006a), aun hoy falta una mayor definición de ciertos aspectos por aclarar.

Si bien es cierta la posición acerca de que la sociología puede ser parte de importantes cambios sociales, brindando valiosos aportes a proyectos sociales para una mayor igualdad y equidad en la sociedad o brindando posibilidades de participación y desarrollo a comunidades y sectores marginados de la toma de decisiones, desafortunadamente en el campo político nacional, en cuanto a la ejecución y gestión de ciertas políticas y decisiones, no son siempre profesionales en sociología (o en educación) quienes deciden el rumbo de la política educativa. 
Es decir, una investigación puede brindar numerosos y valiosos aportes de importancia para la sociedad, pero si no cuenta con la aceptación y el "aval" de sectores políticos, es posible que no se materialice su puesta en práctica.

Por otro lado, de igual forma como se sugirió el objeto de estudio de la sociología de la educación, de forma propositiva se puede reconocer que lo que caracteriza a una investigación de esta rama de la sociología, además de su objeto de estudio, es que toma como paradigma algunas de sus perspectivas principales (positivismo, fenomenología), emplea los métodos propios de la sociología y diseña una metodología científicamente adecuada para el estudio de la educación, con el fin de generar conocimiento sobre la realidad de una sociedad, comunidad, grupo, individuos, sistema, institución, etc.

Vale reconocer que es necesario profundizar en el debate y en la proposición de la delimitación clara del campo de estudio de la sociología de la educación, al igual que en el desarrollo de métodos propios acordes con su objeto de estudio. En otras palabras, es claro que el debate teórico no acaba aquí.

Conviene darle una continuidad en tanto ha sido precisamente este debate lo que ha dado como resultado una variedad de corrientes teóricas y de aportes conceptuales importantes. Así por ejemplo, Van Haecht (1999) toma distancia de los autores clásicos, pero considera su influencia en la sociología y señala que esto ha dado pie al surgimiento de "la sociología "crítica" de la educación" como el esquema de la reproducción (Bourdieu y Passeron), la teoría de la desventaja sociocultural y lingüística (Bernstein), el individualismo metodológico (Boudon), etc., pero aún queda pendiente el debate sobre los posibles métodos para la sociología de la educación.

Finalmente, luego de establecidas las relaciones sociedad-educación-Estado- sistema educativo-mercado, falta también indicar algunos de los posibles temas y problemas por estudiar desde la sociología de la educación (ver figura 2).

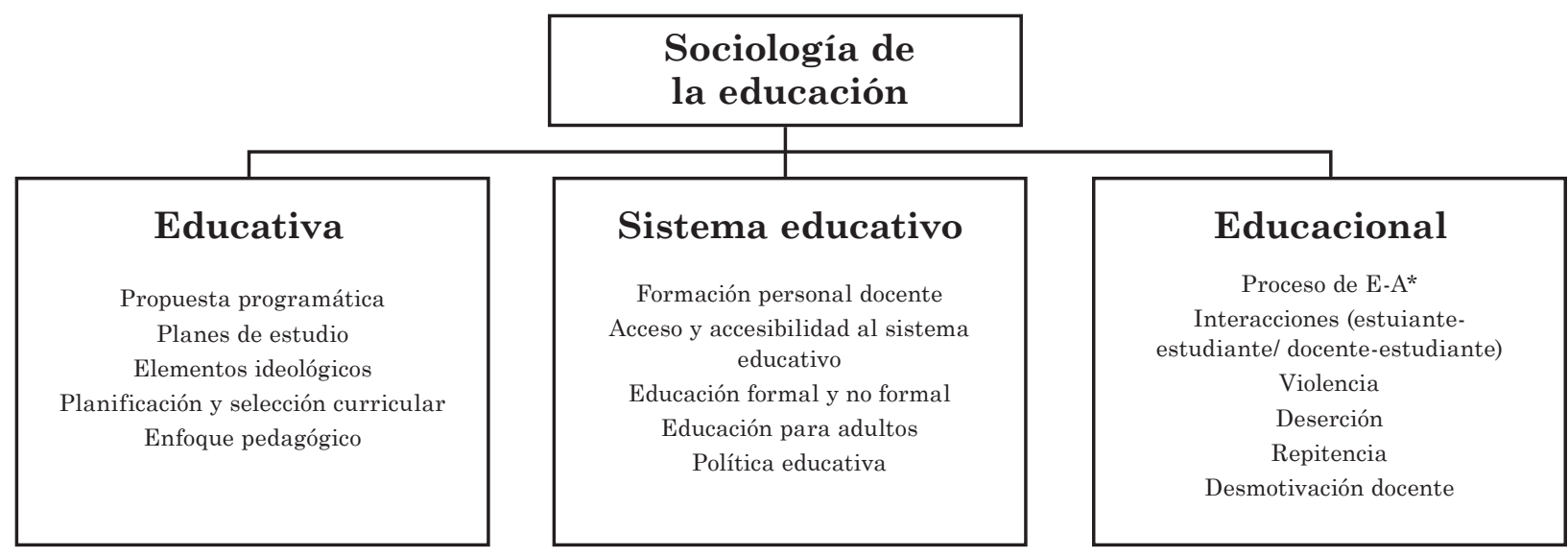

Figura 2. Líneas de investigación de la sociología de la educación y posibles temas por investigar

*proceso de enseñanza y aprendizaje

Nota: Elaboración propia. 
Dentro de cada línea investigativa, se sugieren temas que podrían ser abordados dentro de la sociología de la educación. Por supuesto, algunas personas se "escandalizarían" con esta propuesta, debido a que toca algunos temas que algunos considerarían "feudo privado" de otras ciencias como la psicología.

Pero, en la época actual en que se incentivan las investigaciones interdisciplinarias y transdisciplinarias y ante temas que se encuentra en zonas de frontera (entre 2, 3 o más ciencias) ¿cabe pensar en "segregacionismos científicos"? o ¿Será mejor abrir espacio para la integración entre disciplinas?

\section{Conclusiones}

Como se observa en el repaso por las tradiciones teóricas, no se puede hablar de "sociología" sino de "sociologías". Esto no atenta contra la unidad de análisis de la sociología (la sociedad), pero permite reconocer que las diversas perspectivas varían en su enfoque.

Además, lo anterior evidencia que la construcción de la sociología como un campo científico, es una constante, de manera que el conocimiento científico de lo que se llama «lo social» resulta difícil de definir debido a la dinámica de cambio de las sociedades y de la sociología misma (Berthelot, 2003).

Es decir, la dinámica y el cambio son parte fundamental de la investigación sociológica en cuanto a su objeto de estudio y parte integral del campo de la sociología. Esas condiciones imponen la constante revisión de las aplicaciones de la sociología y de sus ramas.

En el caso de la sociología de la educación, es notorio que no existe una única línea investigativa a la cual todos los autores, llamados clásicos, recurrieran o que permitan definir con claridad el objeto de estudio de la sociología de la educación.

Grosso modo, el énfasis de las diversas teorías mencionadas se sitúan según el mayor o menor énfasis que se le dé dentro de los extremos de las relaciones: microsocial-macrosocial, individuosociedad y acción-estructura. Donde, en algunas de ellas se encierra, hasta cierto punto, algunos de los debates filosóficos de siglos pasados (materialismo-idealismo, racionalismo-empirismo, etc.).

Por eso, se examinó aquellos aspectos que sirvieran para definir el objeto de estudio de la sociología de la educación. De forma secundaria, este documento sirve como un repaso introductorio para toda aquella persona que desee comenzar a explorar dentro del campo y desconoce parcial o totalmente, a los autores más representativos de las escuelas teóricas reseñadas.

Por otro lado, se puede concluir que la realidad educativa es plural, porque no sólo se restringe a la escuela y el colegio, pero desafortunadamente en muchas definiciones de la educación se reduce esta a la educación formal.

Ante esto se vuelve necesario reflexionar respecto a algunas incógnitas tales como: ¿si se podría generarse una sociología de la educación no formal? ¿Una sociología de las personas o grupos con necesidades educativas especiales?, entre otras, con el fin de desarrollar con mayor detalle el alcance de la sociología en cuanto a la interpretación y explicación del fenómeno educativo, como forma crítica de la educación, el sistema, el pensamiento y como posible generadora de soluciones. 
Por eso, siguiendo la óptica de Jensen (1971) cabría preguntarse ¿será acaso que la sociología de la educación no podrá aportar para la comprensión y búsqueda de soluciones a problemas como la violencia en centro educativos (estudiante a estudiante/ estudiante a docente), la deserción, la repitencia, el llamado fracaso escolar (que comúnmente se le endilga al estudiante sin reflexionar sobre las posibles causas sociales involucradas), etc.?, es decir, pensar sobre problemas relacionados con la práctica educativa en términos prácticos, de manera que esta no sólo se restrinja al proceso de enseñanza y aprendizaje.

Por supuesto, al leer las sugerencias previas para tratar de definir el objeto de estudio de la sociología de la educación al igual que los temas sugeridos en el esquema 2, se quiere destacar que al ser la educación un macrotema, la tradicionalmente llamada sociología de la educación (relación sociedad-educación-sociedad), engloba la sociología educacional, la sociología educativa y del sistema educativo, resultando ser líneas de investigación de un mismo tema, pero su categorización facilita la especificación de diversos aspectos dentro de un mismo objeto de estudio, que pueden incluirse dentro de lo que se llamará sociología de la educación, más que el resultado irreconciliable de un debate sin fin.

Por último, también se pretende llamar la atención sobre los aportes que algunas ciencias (como la sociología) pueden dar en diversos campos y las posibles integraciones o complementariedades teóricas o metodológicas que puedan surgir, alrededor de un tema como la educación, que es viable abordar desde una óptica inter y transdisciplinaria.

En ese sentido es claro que existe la necesidad ineludible de mayores investigaciones, artículos y ensayos, ya que aún falta mucho por explorar y desarrollar, tanto dentro de este campo científico, como dentro de la educación como objeto de estudio. Se exhorta pues al lector y lectora a que ahonde en el desarrollo de propuestas y proyectos investigativos y de esta manera aporte también al debate respecto a la sociología de la educación.

\section{Referencias bibliográficas}

Adorno, T. (2006). Introducción a la sociología. España: Editorial Gedisa.

Adorno, T. y Horkheimer, M. (1969). La sociedad. Lecciones de sociología. Argentina: Editorial Proteo.

Althusser, L. (2008). Ideología y aparatos ideológicos de Estado. México: Grupo Editorial Tomo.

Álvarez-Uría, F. (2007). Referencias biográficas y bibliográficas de Émile Durkheim. En: ÁlvarezUría, F. (ed.). Karl Marx, Max Weber y Emile Durkheim. España: Ediciones Morata S. L.

Berthelot, J. M. (2003). La construcción de la sociología. Argentina: Ediciones Nueva Visión.

Bourdieu, P. y Passeron, J. C. (2006). Los herederos. Argentina: Siglo XXI Editores.

Díaz, C. (2009). Viejas y Nuevas Ideas en Educación. Una historia de la pedagogía. España: Editorial Popular. 
Dubet, F. (2006). La Escuela de las Oportunidades ¿Qué es una escuela justa? España: Editorial Gedisa.

Durkheim, E. (1980). La Evolución Pedagógica en Francia. En: Gras, A. (comp.). Sociología de la educación. España: Narcea S. A. de Ediciones.

Durkheim, E. (2002). La Educación Moral. España: Ediciones Morata S. L.

Durkheim, E. (2007). El papel de las Universidades en la educación social del país. En: Álvarez-Uría, F. (ed.). Karl Marx, Max Weber y Emile Durkheim. España: Ediciones Morata S. L.

Durkheim, E. (2007). La escuela del mañana. En: Álvarez-Uría, F. (ed.). Karl Marx, Max Weber y Emile Durkheim. España: Ediciones Morata S. L.

Durkheim, E. (2007). Debate sobre la Educación Sexual. En: Álvarez-Uría, F. (ed.). Karl Marx, Max Weber y Emile Durkheim. España: Ediciones Morata S. L.

Durkheim, E. (2009). Educación y Sociología. España: Editorial Popular.

Elias, N. (2002). Compromiso y distanciamiento. España: Ediciones Península.

Gardou, C. (2000). La educación especial. En: Avanzini, G. (coord.). La pedagogía hoy. México: Fondo de Cultura Económica.

Gómez, C. y Domínguez, J. (2001). Sociología de la Educación. Manual para maestros. España: Ediciones Pirámide.

Gras, A. (1980). Sociología de la educación. España: Narcea S. A. de Ediciones.

Horkheimer, M. (2000). Teoría Tradicional y Teoría Crítica. España: Ediciones Paidós Ibérica.

Janne, H. (1980). Prólogo. En: Gras, A. (comp.). Sociología de la educación. España: Narcea S. A. de Ediciones.

Jensen, G. (1971). Sociología educacional. Argentina: Editorial Troquel.

Kupiec, A. (2008). Karl Mannheim ideología, utopía y conocimiento. Argentina: Ediciones Nueva Visión.

Lahire, B. (2006a). El espíritu sociológico. Argentina: Ediciones Manantial SRL.

Lahire, B. (2006b). ¿Para qué sirve la sociología? Argentina: Siglo XXI Editores. 
Le Bouëdec, G. (2000). La educación de los adultos. En: Avanzini, G. (coord.). La pedagogía hoy. México: Fondo de Cultura Económica.

Marx, K. (1997). Manifiesto del Partido Comunista. México: Editores Mexicanos Unidos.

Marx, K. (2007). Trabajo de los niños y escolarización. En: Álvarez-Uría, F. (ed.). Karl Marx, Max Weber y Emile Durkheim. España: Ediciones Morata S. L.

Marx, K. (2007). Exámenes y Burocracia. En: Álvarez-Uría, F. (ed.). Karl Marx, Max Weber y Emile Durkheim. España: Ediciones Morata S. L.

Ottaway, A. K. C. (1965). Educación y sociedad. Introducción a la sociología de la educación. Argentina: Editorial Kapelusz.

Simmel, G. (2008). Pedagogía Escolar. España: Editorial Gedisa.

Taberner, J. (2005). Sociología y Educación. El sistema educativo en sociedades modernas. Funciones, cambios y conflictos. España: Editorial Tecnos.

Touraine, A. (2009). La mirada social. Un marco de pensamiento distinto para el siglo XXI. España: Ediciones Paidós Ibérica.

Van Haecht, A. (1999). La escuela va a examen. Preguntas a la sociología de la educación. Argentina: Editorial Biblos- Miño y Dávila Editores.

Vernik, E. (2008). Posfacio: Simmel como pedagogo. La educación como vida. En: Simmel, G. Pedagogía Escolar. España: Editorial Gedisa.

Watier, P. (2005). Georg Simmel sociólogo. Argentina: Ediciones Nueva Visión.

Weber, M. (2000). El político y el científico. España: Alianza Editorial.

Weber, M. (2008). Economía y Sociedad. México: Fondo de Cultura Económica.

Weber, M. (2007). Las universidades americanas y las alemanas. En: Álvarez-Uría, F. (ed.). Karl Marx, Max Weber y Emile Durkheim. España: Ediciones Morata S. L.

Weber, M. (2007). La pretendida libertad intelectual en las universidades alemanas. En: ÁlvarezUría, F. (ed.). Karl Marx, Max Weber y Emile Durkheim. España: Ediciones Morata S. L.

Weber, M. (2007). La libertad intelectual en las universidades. En: Álvarez-Uría, F. (ed.). Karl Marx, Max Weber y Emile Durkheim. España: Ediciones Morata S. L.

Whitty, G., Power, S. y Halpin, D. (1999). La escuela, el estado y el mercado. España: Ediciones Morata S. L. 